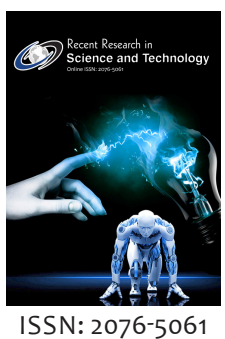

*Corresponding Author: Taslima Binte Hossain Email: hossaintas@gmail.com

\section{Voice mail application for visually impaired persons}

\author{
Taslima Binte Hossain ${ }^{a *}$, Yeasmin Ara Akterb, Md. Ataur Rahmanc \\ aDepartment of Computer Science and Engineering, Port City International University, Nikunja Housing Society, \\ South Khulshi, Chittagong,4202, Bangladesh, 'b School of Science, Engineering and Technology, Department \\ of Computer Science and Engineering, East Delta University, Abdullah Al Noman Road, Noman Society, East \\ Nasirabad Khulshi, Chittagong, 4209, Bangladesh, 'University of Saarland, Department of Computational \\ Linguistics, Saarbucken, Germany
}

\begin{abstract}
Technology-based applications are developed day by day in the whole world. But more of those are unusable for the visually impaired people. One of such applications, email has become an important and significant part of formal communication in the professional world. Thus, Voice Mail turns out to be an application through which visionless people can easily send or receive emails. The application uses "Voice to Text" and "Text to Voice" conversion to be usable for the visually impaired people. The system at first takes voice signals from the users as inputs, processes the signals and then converts that voice signals into the text. After that a text processing method is applied to convert the text into voice. Finally, the gmail server is used to connect the server with generating address to send or receive any mail. This application is useful for not only blind people but also for the normal people who always remain busy and doesn't have time to type in keyboard. Moreover, this application is very much helpful and efficient in time utilization.
\end{abstract}

KEYWORDS: VMS, voice to text, text to voice, voice signals, voice mail

\section{INTRODUCTION}

Now a days, internet has become an inevitable part of our day to day life since most of the mobile applications run through internet. One of major applications is Email. Although, a lot of people can't use this application because of their blindness. To solve their problem, we have implemented a voice mail application which is accessible by voice and this application doesn't need any vision to be executed. Applications which are used for send or receive mail by using only voice that is called "Voice mail system". It'll help the visually impaired people to use an email system more effectively and efficiently by gibing the input by their voice. The system processes user's voice inputs to use a mail system e.g. Gmail account.

Several voice mail applications has already been made by scholars at home and abroad [1][2][3][4][5]but most of them drawbacks such as no instructions for blind people with voice, some system doesn't repeat the email address after getting input, some system doesn't have the inbox options etc. These lacking are overcome in our system.

\section{SYSTEM STRUCTURE AND MODULES}

Our system is a voice mail android application rather than desktop application by which visionless people can easily transfer email through their voice. To make the application, several processing has been employed to transfer the Voice Signals to gmail server. They are i) Process the Voice using API ii) Convert to Text iii) Process the text using API iv) Convert to Voice v) Connecting to the server using Gmail. API.

This project is designed using some set of APIs:

- Google Speech API- This API is used to convert the audio into text by applying powerful neural network models. This API recognize audio uploaded in the system and integrate with user's audio storage on Google Cloud Storage.

- Google Text to Speech API- It takes a text stream and sends a request to Google Translate API Web servers to return an audio file with text converted into speech.

- Gmail API- This API gives the advantage of accessing Gmail mailboxes and send mail. Most of the programmers give highest priority to the Gmail API for authorized access to a user's Gmail data.

Copyright: $\odot$ The authors. This article is open access and licensed under the terms of the Creative Commons Attribution License (http://creativecommons.org/licenses/by/4.0/) which permits unrestricted, use, distribution and reproduction in any medium, or format for any purpose, even commercially provided the work is properly cited. Attribution - You must give appropriate credit, provide a link to the license, and indicate if changes were made. 


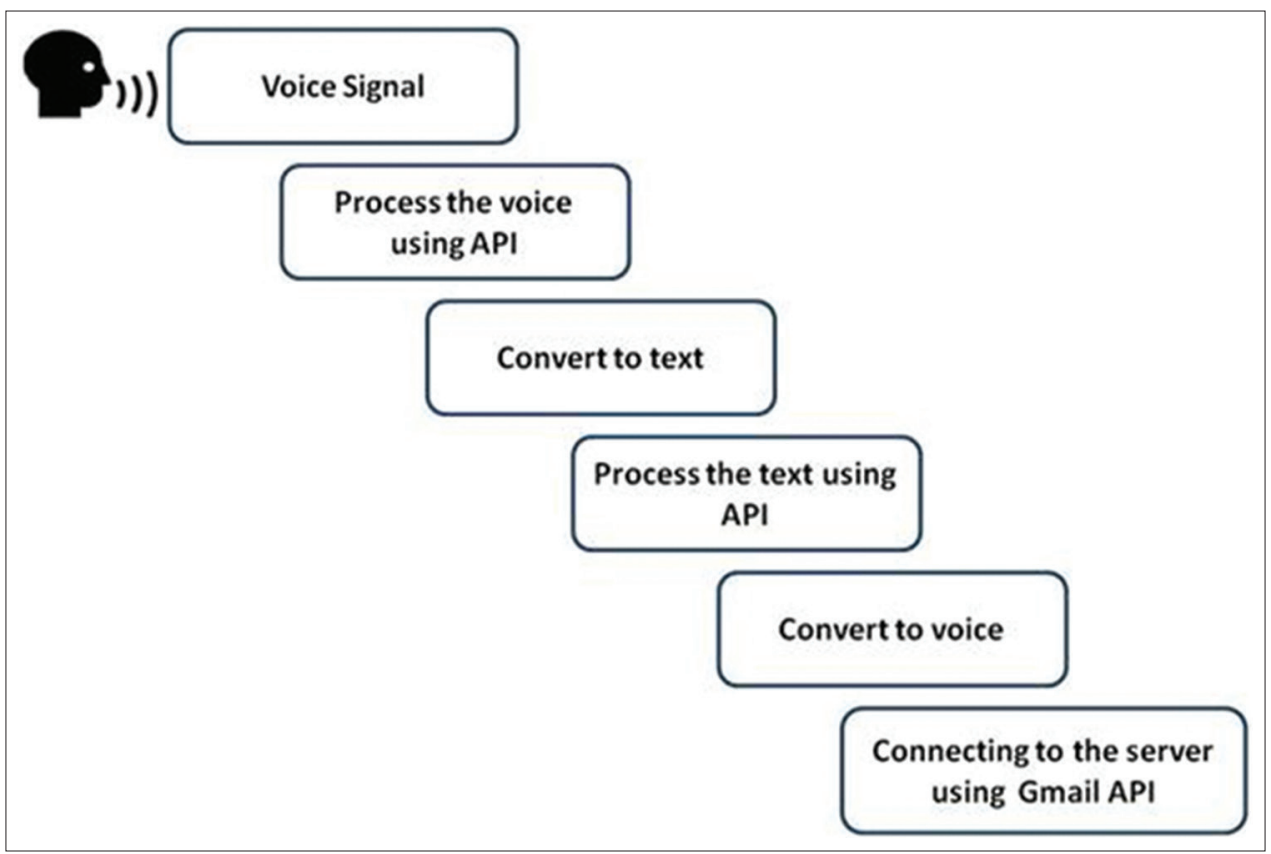

Figure 1: System implementation process with APIs

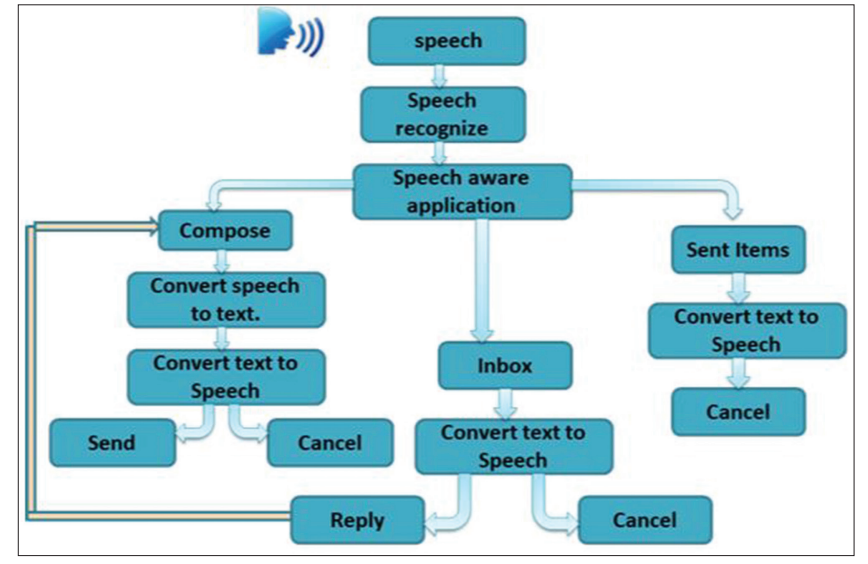

Figure 2: Implementation modules of the system

The system implementation processes and modules on the APIs are shown in Figures 1 and 2. When voice is given as input by the user then it call the google speech to text conversion API and match the logical instruction. After that, text to speech conversion API process the converted text and then call the Gmail API to transfer the text to gmail address that is provided by the user.

\section{SYSTEM DESIGN AND IMPLEMENTATION}

Our VSmail application has been developed and implemented by using JAVA apks and Google APIs. So to use the VSmail, user need to login account in the android phone setting, then it will synchronize with this application. After opening the application, user needs to tap on the screen to listen the instruction what to do next. The instruction is to input voice to choose any one of three categories as Figure 3a) which are

- send email

- inbox and

- sent items
The option to input voice is displayed as Figure $3 \mathrm{~b}$. If input voice is unrecognized, the system gives instruction to tap the screen for again input voice as Figure3c. If input voice is recognized as send mail, then system alert to the user that the next step is send email as Figure3d.

\section{Send Email Module}

With the VSmail, users can send email to other email users. If anyone say send, then VSmail will take the user to send email module which has three steps:

- Address: System gives instruction how to input receiver's email address. User can give input address sentencewise or letterwise. For the accuracy they should input the voice letter by letter and after that tap to the upper half to input domain name. (Figure 4)

- Subject: The second step is giving the subject as voice input as all the gmail user needs to insert a subject. (Figure 5).

- Compose Email: Last step is email body. User can input the actual email saying the sentences in English. After the completion of giving input, system will read aloud the inputted voice to make sure whether the email body is correct or not. So, user can identify what sentences are recognized by the system. (Figure 6). A figure of successfully sent mail to receiver is shown in Figure 7.

\section{Inbox Module}

One of the categories of VSmail is Inbox. Here user can select this category by saying 'Inbox' and can hear the previous emails. If the system recognizes input voice as inbox, then it goes to inbox of the mail server (Figure 8). User can do the following tasks in this option:

- Users can make emails read or unread. If user wants to hear the email, the need to say "Open" instruction or doesn't want to hear the email then they need to say "Next" instruction as voice input. 


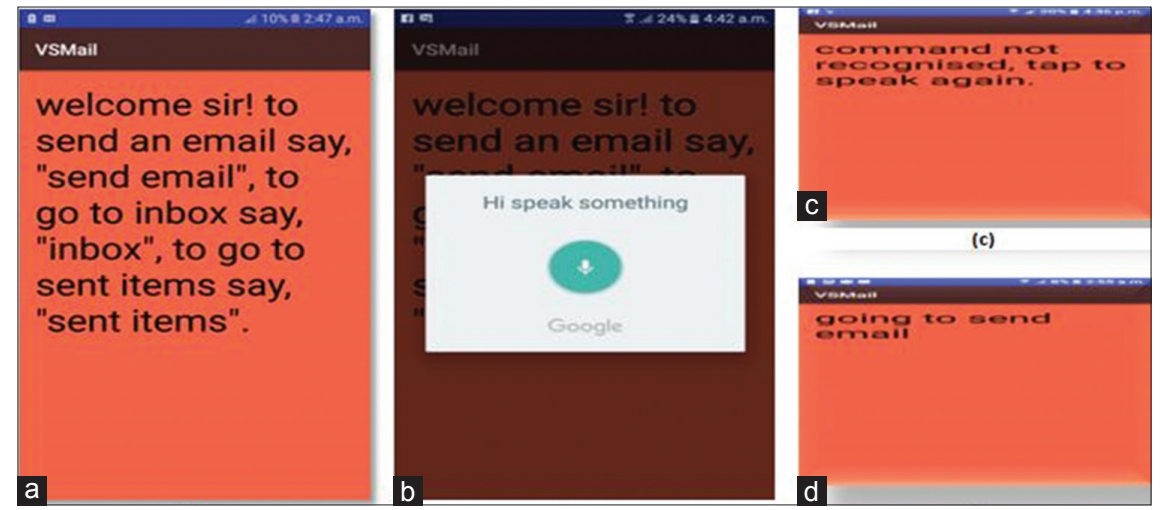

Figure 3: a) Categries of the system b) Option to input voice c) When input is unrecognised d) When input is recognise

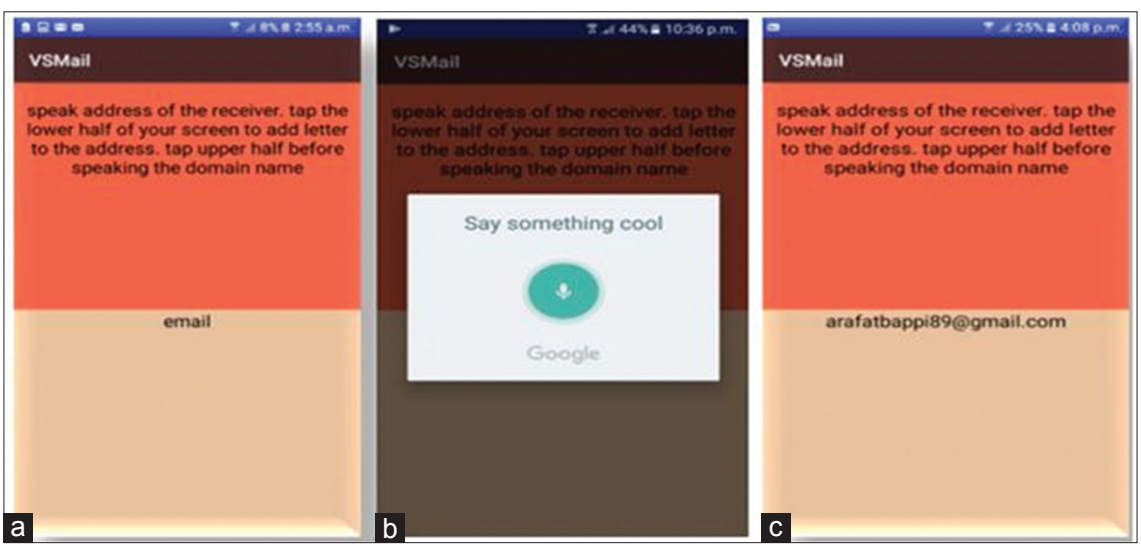

Figure 4: a) Address page of the system b) Input a address letter by letter c) Complete email address taken by system

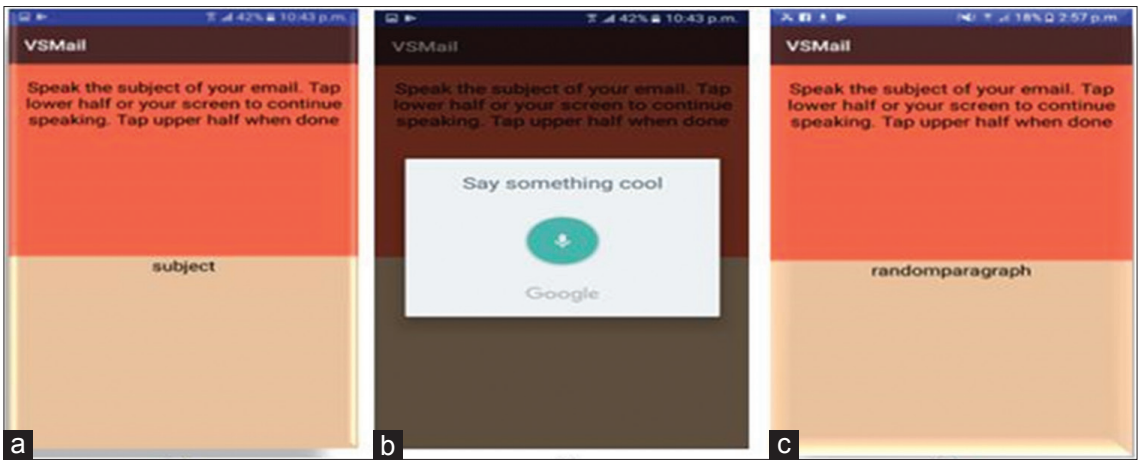

Figure 5: a) Subject page of the system b) Voice input of the email subject c) Complete email subject
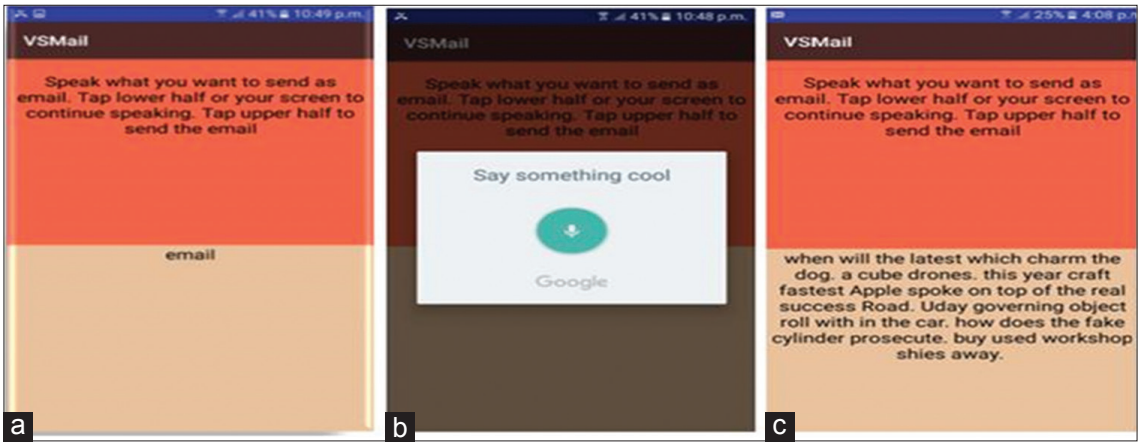

Figure 6: a) Email body page of the system b) Voice input of the email body c) Complete email body 


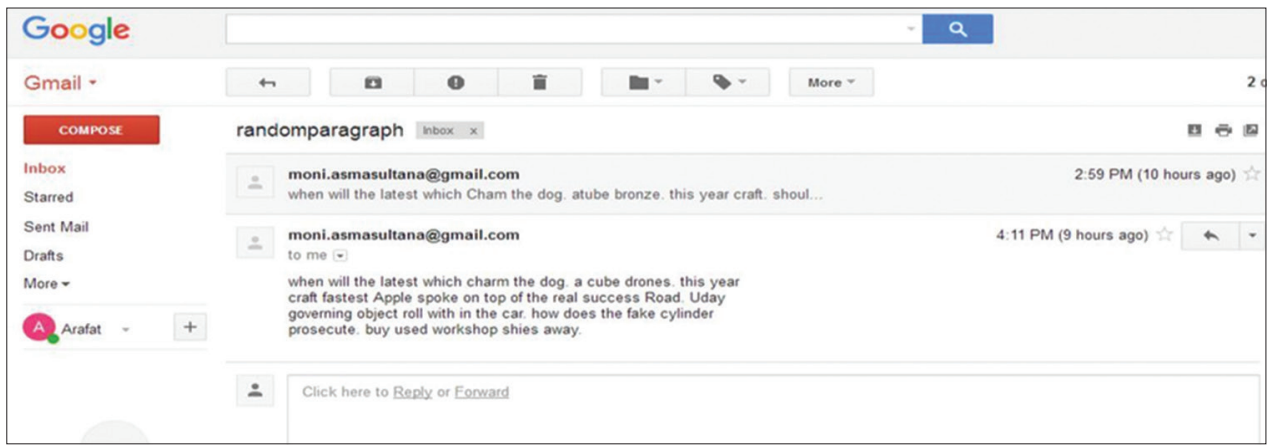

Figure 7: Successfully sent mail to the receiver
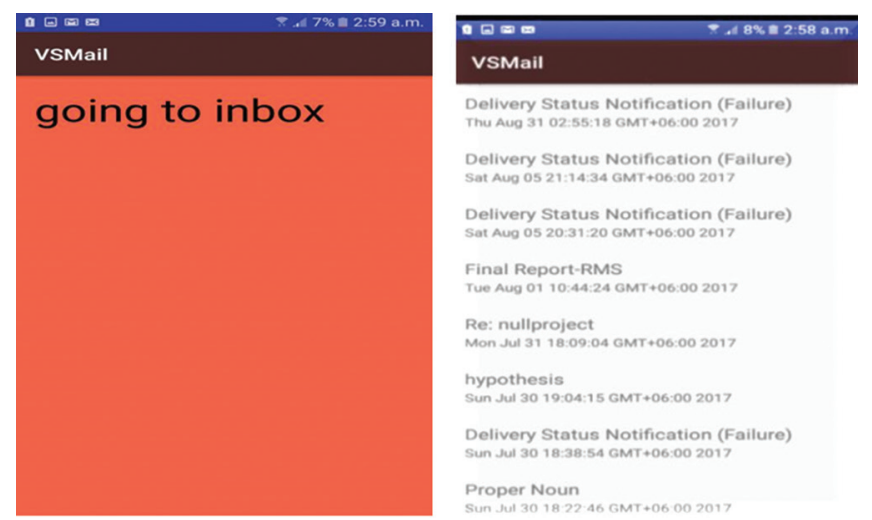

Figure 8: Recognized input voice as Inbox

- After hearing the email, users can reply email to receivers. In this case, users don't need to input an email address. It will start from the subject.

\section{Sent Items}

Users can hear all delivered emails in this section using the "Open" instruction to hear the sent email or "next" instruction to go to the next sent mail.

\section{CONCLUSION AND FUTURE WORK}

The voice mail application can be used easily and efficiently by a blind person to access mails. Thus reliance of visually impaired persons on other people for their activities related to mail can be reduced. Since, right now our system can't take voice signals in Bangla, our future plan is to make the system more robust so that blind people can use the system with Bangla speech.

\section{REFERENCES}

1. Jagtap Nilesh, P. A. C. S. a. B. M., 2014. Voice Based System in Desktop and Mobile Devices for Blind. International Journal of Emerging Technology and, 4(2), pp. 404-407.

2. Pranjal Ingle, H. K. A. L., 2016. Voice based e-mail System for Blinds. International Journal of Research Studies in Computer Science and Engineering, 3(1), pp. 25-30.

3. Rahul Anwani, U. S. R. R., 2015. VMAIL: Voice Based Email Application. International Journal of Computer Science and Information Technologies, 6(2488-2490).

4. Shabana T., A. A. R. A. A. K., 2015. Voice Based Email System for Blinds. International Journal of Advance Foundation And Research In Science \& Engineering, 1(10).

5. Ummuhanysifa U., N. B. P. K., n.d. Voice Based Search Engine and Web page Reader. International Journal of Computational Engineering Research, pp. 1-5. 